\title{
Lidil
}

Revue de linguistique et de didactique des langues

$44 \mid 2011$

Langues, minor(is)ations et marginalisations

\section{Langues, minor(is)ations, marginalisations : une image de la linguistique?}

\section{Louis-Jean Calvet}

\section{OpenEdition}

\section{Journals}

Édition électronique

URL : http://journals.openedition.org/lidil/3149

DOI : 10.4000/lidil.3149

ISSN : $1960-6052$

\section{Éditeur}

UGA Éditions/Université Grenoble Alpes

\section{Édition imprimée}

Date de publication : 15 décembre 2011

Pagination : 137-144

ISBN : $978-2-84310-212-7$

ISSN : 1146-6480

\section{Référence électronique}

Louis-Jean Calvet, "Langues, minor(is)ations, marginalisations : une image de la linguistique ? », Lidil [En ligne], 44 | 2011, mis en ligne le 15 juin 2013, consulté le 19 avril 2019. URL : http:// journals.openedition.org/lidil/3149; DOI : 10.4000/lidil.3149 


\title{
Postface
}

\section{Langues, minor(is)ations, marginalisations : une image de la linguistique?}

\author{
Louis-Jean Calvet*
}

Ce numéro, malgré son titre, Langues, minor(is)ations et marginalisations, qui lui donne un aspect très pointu, m'avait été oralement présenté par Marielle Rispail et Stéphanie Clerc, lorsqu'elles m'ont demandé de rédiger cette postface, comme une sorte de bilan fait par de jeunes chercheurs sur la sociolinguistique aujourd'hui. La lecture de l'appel à contributions nous montre d'ailleurs que sur ce point le projet était clair, annonçant que ce numéro traiterait

de la sociolinguistique et des usages langagiers. Il a pour ambition de faire le point sur des recherches récentes et inédites, dans le domaine sociolinguistique, entendu comme l'étude du rapport des langues avec ceux et celles qui les parlent, ainsi que des langues entre elles, des enjeux qui sous-tendent ces rapports et de leurs implications sur divers terrains sociaux où se s'engagent et s'impliquent les chercheurs. Cet état des lieux prendra en compte les évolutions linguistiques, les dynamiques langagières, les éventuels conflits ou tensions, qui agitent la vie des langues dans le monde, à un niveau macro ou micro - de l'étude de cas aux questionnements de politique linguistique.

À l'arrivée, le résultat est évidemment différent puisque sur les huit textes qui le composent cinq au moins portent sur la question de langues minoritaires ou minorées, et c'est évidemment la moisson des textes proposés qui a finalement déterminé le numéro. Comme un avion détourné par ses passagers qui décident d'une autre direction, le numéro a ainsi changé de cap. Il est donc intéressant de réfléchir sur la traduction effectuée par les contributeurs de cette thématique initiale : un appel à contribution sur « la sociolinguistique et les usages langagiers » débouche sur

* Université de Provence. 
un numéro dans lequel sont majoritairement évoquées des situations comme celles des Grecs turcophones, de la diglossie en Corse, du francique en Lorraine... Nous avons là une sorte d'indice : la sociolinguistique, les usages langagiers renvoient pour la plupart des auteurs retenus ici aux langues minoritaires ou minorées. Et une recherche rapide nous montre que la notion de communauté apparait dans sept articles sur neuf tandis que celles de politique (politique linguistique, glottopolitique) et d'identité (nationale, plurielle, linguistique) apparaissent dans six articles : communauté(s), politique, identité pourraient être les mots clés de ce recueil.

Un autre point de vue intéressant est celui qui prend en compte les références bibliographiques et théoriques des auteurs. En excluant les autocitations (les auteurs se citant eux-mêmes) et les auteurs cités une seule fois ou dans un seul article, nous pouvons extraire de la bibliographie des huit articles le «palmarès » suivant, dans lequel on soulignera la prédominance d'auteurs français :

- W. Labov, cité dans quatre articles pour deux ouvrages (Sociolinguistique, Language in the Inner City) et deux articles;

- P. Bourdieu, cité dans trois articles pour deux ouvrages (Ce que parler veut dire, Le sens pratique);

- $\quad$ L.-J. Calvet, cité dans trois articles pour quatre ouvrages (Linguistique et colonialisme, Pour une écologie des langues du monde, La guerre des langues, Les voix de la ville);

- $\quad$ F. Gadet, citée dans trois articles pour un ouvrage (La variation sociale en français);

- J.-B. Marcellesi, cité dans deux articles pour quatre articles.

J'ai souligné que la majorité des articles analysait des situations de domination linguistique ou de diglossie, il faut y ajouter ceux qui décrivent celles des femmes maghrébines ou de jeunes Rouennais : l'image de la sociolinguistique qui ressort de ce numéro est sur le plan conceptuel, celui des références théoriques, assez classique (Labov, Bourdieu, Calvet...) mais sur le plan des thématiques ce qui s'impose est l'idée de situations. En marge de, ou en écho à, ce numéro, je voudrais donc tenter de réfléchir un peu sur cette notion.

Lénine, dans sa Maladie infantile du communisme, le gauchisme, prônait « l'analyse concrète d'une situation concrète », ce qui était pour lui « l'âme vivante du marxisme ». Cette formule semble tout d'abord 
frappée au coin du bon sens : ne faut-il pas mieux en effet analyser des situations précises plutôt que de raisonner sur des chimères ou des bases erronées? Mais en réfléchissant un peu, on se dit que la formule présuppose l'existence d'une réalité (la « situation concrète ») qu'il suffirait d'étudier de façon adéquate (par une « analyse concrète ») pour y voir clair et savoir où aller politiquement. Et c'est là que commencent les problèmes. Cette formule contient en effet un risque : celui de considérer comme réalité, comme situation concrète, une illusion commode qui nous convient pour mener nos analyses, ou une fiction utile qui les justifie par avance. Mais surtout, elle pose un problème logique : pour partir d'une situation concrète, soit l'on considère qu'elle est donnée, qu'elle se trouve devant nous, et qu'il suffit donc de l'analyser, ce qui est un peu naï, soit l'on considère qu'il faut la construire. Et, pour la construire, il faut partir d'un point de vue, avoir des critères de pertinence, c'est-à-dire qu'il nous faut analyser avant d'analyser, et la théorie, à ce jeu, risque fort de se mordre la queue : aucune situation n'est immédiatement préhensible, il faut la construire, et cette construction implique une forme de téléologie.

Je voudrais passer au filtre de ce programme, analyse concrète d'une situation concrète, la question de la description linguistique, et les problèmes qu'elle me (nous) pose, étant entendu que pour moi cette description ne peut être uniquement interne mais qu'elle devrait prendre en compte le contexte social et historique dans lequel se situent les actes de communication et les effets de sens. Joshua Fishman, dans un article publié en 1965 dans la revue française La Linguistique avait proposé un programme de description « sociolinguistique » qui était parfaitement résumé par son titre : «Who speaks what language to whom and when? » Il proposait par ces différentes questions une pluralité de points de vue (auxquels nous devrions d'ailleurs ajouter deux autres questions qui manquent cruellement: why et where), mais impliquait surtout l'existence de langues aisément identifiables: what language, «quelle langue ». Or les choses ne sont peut-être pas aussi simples, la notion de langue n'est peut-être pas aussi évidente et dès lors l'idée de situation linguistique concrète est peut-être plus difficile à manier qu'il n'y parait.

Qu'est-ce en effet qu'une situation linguistique concrète ? Je commence ce texte en août 2011 à Kairouan, en Tunisie. Nous sommes au deuxième jour du ramadan, des phrases en arabe classique diffusées par les haut-parleurs des mosquées rythment la journée, les employés de l'hôtel dans lequel je suis descendu échangent en arabe tunisien, certains 
d'entre eux parlent un français parfait, d'autres le comprennent à peine, et les femmes de ménage qui font les chambres ne parlent qu'arabe tunisien. Dans les rues de la ville, dans les souks, on entend le même arabe local et la connaissance du français est en gros fonction de l'âge : les vieux le parlent mieux que les jeunes, ce qui témoigne des successives réformes de l'enseignement qu'a connues le pays... Ce rapide tableau se complexifierait encore plus si nous prenions en compte l'environnement graphique (deux alphabets, l'arabe et le latin, servant à transcrire plusieurs langues, essentiellement arabe standard et français, et dans une moindre mesure anglais) et le rapport à l'écriture : qui lit et écrit quoi ? Quelle forme d'arabe ? Ainsi un ami d'une soixantaine d'années, dont l'arabe tunisien est la langue maternelle, m'explique qu'il ne lit la presse qu'en français : "Lorsqu'il faut vraiment que je consulte un article en arabe, je le lis trois ou quatre fois à voix haute pour arriver à le comprendre...»

Déplaçons-nous de quelques dizaines de kilomètres vers l'est pour aller à Sousse, au bord de la mer, ville touristique offrant de nombreux hôtels internationaux. On y retrouve l'arabe tunisien, l'arabe standard, le français ainsi que quelques autres langues étrangères, italien, allemand, anglais... Déplaçons-nous encore, de quelques dizaines de kilomètres vers le nord cette fois-ci. À Tunis l'environnement graphique est le plus souvent bilingue, arabe et français, et la pratique du français est plus présente que dans le centre du pays. Ajoutons à cela que la télévision italienne est souvent captée en Tunisie, qu'une partie des migrants s'y rend en quête de travail, et que la langue italienne a un statut particulier explicable à la fois par le passé (la communauté italienne importante à l'époque du protectorat français) et par le présent.

Le lecteur comprendra aisément qu'en essayant de déterminer la situation linguistique concrète dans laquelle je me trouvais le 2 août 2011 j'ai dû classer, étiqueter (arabe standard, arabe tunisien, français courant ou pas, italien, langues étrangères...), c'est-à-dire proposer une typologie et en fait analyser la situation avant même de l'analyser. En outre j'ai oblitéré de nombreuses choses, tout ce qui relève des sentiments linguistiques, des représentations, qui en l'occurrence pourraient aller d'une mythification d'une langue «pure » et « sacrée », l'arabe classique, à la haine du français, héritage de la colonisation, ou encore, exactement à l'inverse, d'une pulsion vers le français, langue « de la démocratie », " de la laïcité », vers un refus de l'arabe, "langue de l'obscurantisme ». Et tout ceci est bien sûr à analyser dans le cadre de la « révolution» de janvier 2011, de la campagne électorale pour élire 
une assemblée constituante, des interrogations sur la place de la religion dans la future constitution, etc. Ce qui me mène à une première question : qu'est-ce qui nous donne la situation concrète que nous nous proposons d'analyser? Qui décide de cette situation concrète ? Comment éviter, comme je l'ai écrit plus haut «d'analyser avant d'analyser », ou de nous donner une présentation de la situation qui soit nous arrange ou soit est produite par notre vision préétablie des faits ?

Les réponses les plus courantes à ces questions peuvent se ramener, de façon générique, à deux notions, celles de terrain et de corpus. Ces deux notions ne renvoient pas exactement à la même chose. Le corpus est un ensemble clos, présumé homogène, dont on va tirer toutes les données sur « la langue » que l'on décrit, ou plutôt sur l'idiolecte que l'on décrit. Il est donc censé assurer la cohérence de la description : ce qui est dans le corpus est retenu, ce qui n'est pas dans le corpus est rejeté ou ignoré. Reste bien sûr à savoir comment l'on constitue ce corpus, mais la linguisterie (Lacan) a depuis longtemps résolu, ou prétendu résoudre, cette question en balisant soigneusement des critères de scientificité. Le seul ennui est qu'un corpus, si large soit-il, ne suffira jamais à représenter une situation complexe comme celle que j'ai rapidement évoquée plus haut, et toutes les situations présentent un degré au moins semblable de complexité. Il nous faut donc nécessairement établir des liens entre ce corpus et la situation dans laquelle il a été recueilli, faute de quoi il ne serait que l'instrument d'une approche fragmentaire, plus pointilliste que pointilleuse, des faits. Il est facile, avec les instruments informatiques dont nous disposons aujourd'hui, de sortir d'un immense corpus (par exemple des millions de pages dans une langue donnée sur le web) des milliers de phrases contenant un fait de langue quelconque, puis de soumettre ces phrases à une quelconque analyse automatique. Mais l'apparente scientificité du mode d'extraction du corpus et de son traitement n'enlève rien à l'aspect le plus souvent parcellaire des thèmes traités. Certains pourront considérer que c'est justement l'accumulation de descriptions parcellaires qui permet d'aboutir à une description globale, ce à quoi l'on répliquera qu'un ensemble est toujours quelque chose de plus que la somme des constituants perçus (on pourrait évoquer ici la gestalt theorie), et qu'en fait nous configurons (" configurer » pourrait être la traduction du verbe allemand gestalten) les différents éléments que nous percevons pour leur donner un sens parmi d'autres possibles. Ainsi parler d'une constellation (Orion, la Grande Ourse, etc.) consiste en fait à sélectionner parmi un grand nombre d'étoiles celles que nous pouvons configurer pour inventer une signification graphique. Voir l'image d'un 
chasseur esquissée par certaines étoiles, avec ses épaules, ses pieds, son baudrier, et la baptiser Orion c'est sélectionner certaines étoiles, en exclure d'autres, et inventer un lien entre celles que l'on a retenues. La meilleure preuve en est que d'autres civilisations ont vu (tracé, inventé) d'autres significations à cet ensemble d'étoiles (un dieu pour les Mayas, un mouton pour les Sumériens, etc.).

Cette sélection téléologique n'est pas une vue de l'esprit. La façon dont l'approche générative, sous ses multiples avatars, sélectionne les énoncés qui confirment sa théorie en constitue un exemple. Et son approche ne peut déboucher que sur l'invention de machines à faire des phrases, alors que le but de l'analyse linguistique ne devrait pas être de décrire des « ensembles» (des «langues ») abstraits de leurs contextes, mais des pratiques sociales dans lesquelles ces « ensembles » circulent, s'entrechoquent de façon parfois aléatoire comme des particules dans un mouvement brownien, se mélangent, convergent ou s'opposent.

Quant au travail de terrain, ou à la linguistique de terrain, il s'agit d'une notion floue et un peu fourre-tout qui permet à la fois de se parer des oripeaux de la scientificité sociologique ou ethnologique et de dire parfois n'importe quoi. Certaines techniques d'enquête permettent certes d'approcher des pratiques, de les quantifier, d'autres, comme l'observation participante, peuvent donner l'illusion de la recherche d'une empathie, d'un refus du surplomb, mais rien de cela ne répond à la question qu'est-ce qu'une situation concrète ? Cela pourrait nous mener à la conclusion que l'idée de situation concrète est à la fois trompeuse et dogmatique, qu'une série d'hypothèses enchâssées que l'on tente de vérifier les unes après les autres serait préférable à l'idée de situation ou d'analyse concrètes, et que les linguistes se situent trop souvent dans le confort d'une position en surplomb. Je conclurais donc volontiers que, dans le cas de la formule de Lénine comme en linguistique, il est illusoire de croire en une bonne, grosse et rassurante « situation concrète ».

Revenons maintenant à ce numéro. On y trouve souligné dans certains des articles qu'il regroupe le fait que les concepts évoluent, que la notion d'insécurité linguistique par exemple a changé depuis Labov (Luc Biichlé) comme a évolué celle de diglossie (Pascal Ottavi) ${ }^{1}$. On y trouve

1. Il faudrait d'ailleurs ici relire l'analyse critique que C. Ferguson lui-même a fait de son concept : «Diglosia Revisired », dans Southwest Journal of Linguistics, vil $10 \mathrm{n}^{\circ} 1,1991$. 
aussi le fait que la notion d'identité linguistique peut renvoyer aussi bien au monolinguisme qu'au plurilinguisme et à la diversité (Céline Jeannot, Sandra Tomc et Marine Totozani), ou encore que la lente disparition du francique comme langue première peut être à la fois le produit de comportements et de représentations individuels (je pense ici à certains textes de Daniel Laumesfeld ${ }^{2}$ ) et de la politique linguistique française. En bref on y trouve des notations et des analyses qui vont un peu dans tous les sens, ce qui est à la fois salutaire (pas d'école, pas de dogmatisme, mais une réflexion pluridimensionnelle) et désespérant (en quoi la sociolinguistique a-t-elle bien pu avancer en France depuis vingt, trente ou quarante ans?). De ce point de vue, Langues, minor(is)ations et marginalisations, le titre de ce numéro de Lidil, pourrait tout à fait convenir à une autre livraison dont le thème serait la linguistique (ou la sociolinguistique, ou la (socio)linguistique) qui traite de « langues » sans toujours pouvoir dire de quoi il s'agit exactement, qui est passée en moins d'un demi siècle du statut de « science humaine pilote » à celui de science marginalisée, minorée, et qui se cherche à travers une pluralité de micro spécialisations.

Cette situation n'est d'ailleurs pas désespérée, du moins si nous considérons que la lumière nait toujours de l'obscurité...

Louis-Jean Calvet Aout-septembre 2011

2. Par exemple D. Laumesfeld, 1996, La Lorraine francique : culture mosaïque et dissidence linguistique, L’Harmattan, Paris, p. 61 : «Aussi étrange que cela puisse paraitre, je me souviens de l'instant précis où le couperet est tombé. C'était exactement le jour où je suis revenu de ma première colonie de vacances avec mon frère. J'avais neuf ans, et quand nous sommes sortis du bus, nous chantions une chanson espagnole [...]. Dans la voiture, mon frère et moi répondons en français aux questions que nos parents nous adressent en platt. Le fait est si étrange qu'il nous éclabousse la conscience [...]. Mon père dit : « Mais vous parlez français maintenant? » Ils sourient - nous aussi. Des sourires jaunes qui cherchent à cacher quelque chose dans leur brèche idiote. Entre nous circule une gêne. Quoi répondre ? Il n'y a rien à dire - sinon à sourire bêtement pour entériner le constat. Nous étions tout d'un coup devenus des étrangers. Pas des étrangers venus d'ailleurs, mas des étranges, des êtres qui ne parlent plus comme des familiers. Des étrangers pour nous-mêmes. » 
